\title{
A Comparison of Cloud Computing Platforms
}

\author{
Ikechukwu Nwobodo \\ School of Architecture, \\ Computing and Engineering University of East London Dockland Campus, \\ London United Kingdom \\ i.nwobodo@yahoo.com
}

\begin{abstract}
Cloud computing concept has overhauled the entire structure representation, required for the implementation of IT Infrastructure. This cloud computing approach is gradually eliminating the existing client server system and grid computing. The capabilities associated with the innovation of cloud computing have given rise to users to develop and share different applications on the internet infrastructure. This has also led to the ability to access data at each computer node anytime and anywhere across computer network without limitation of resource requirement or hardware demands. This paper explores the underlying platform that is used by computer architects to design and module cloud computing in order to satisfy the usage requirement. Existing cloud computing module and services can influence and shape the future of IT infrastructure and their development. A comparison of four cloud computing platform namely: AbiCloud, Eucalyptus, Nimbus, and OpenNebula cloud will be presented.
\end{abstract}

Keywords-virtualization; iaas, saas, paas; VM; cloud computing.

\section{INTRODUCTION}

Cloud computing has transformed the representative outlook, which distributed computing such as grid and server client computing present in IT environment. Cloud computing has given birth to a new creative meaning to distributed and off-premises computing. Nwobodo, et al. (2014), defined cloud computing as an on-demand service model for provision of IT, normally based on virtualisation technologies and distributed computing. Cloud computing innovation enable on-demand access to network pool of shared computing resources such as servers, storage, and applications. This model undoubtedly envisage a world where components can be quickly orchestrated, implemented, provisioned, possibly decommissioned and on request scaled up and down to provide utility on-demand model of consumption and allocation.

Ameen, et al. (2012), states that Cloud computing to users, is Pay-Per-Use on demand mode to access expediently shared IT resources via the internet. Cloud resources include applications, services, network and storage and these resources can be deployed easily in a short pace, therefore minimizing interaction and management to the cloud providers. The availability of IT resources has been tremendously improved by cloud computing, making it to own outstanding advantage over other computing techniques. Users have the opportunity of self help services without the need for any physical interaction with the service providers.

Cloud computing resources are transparent to users, meaning that they can dynamically lease and use virtual resources without the need of understanding where and how resources exist. Moreover, all cloud resources can be elastically and quickly deployed. Furthermore, the ability to access data at each computer node anytime and anywhere across computer network without limitation of resource requirement or hardware demands is much beneficial to cloud computing users. (Peng et al., 2009).

\section{Cloud COMPUTING Models AND SERVICES}

The most clearer and broad expression of cloud computing model and classification was made by NIST (2013), where cloud was described as a model of expedient using efficient computing resource, pointing out on four deployment models such as: public, private, hybrid and community cloud. The cloud computing services can be generally categorised into three types: Infrastructure as a Service (IaaS), Platform as a Service (PaaS) and Software as a Service (SaaS) (Lin and Chen 2012).

\section{A. Cloud Computing Model}

The Public cloud model is defined as a cloud computing infrastructure used by third party provider to provide services to different categories of clients via the internet. The infrastructure provided can be worked on simultaneously by several enterprises. Moreover, it is possible to set up the model in multiple data centres operated and managed by third party providers (Sultan, 2013; Kahn et al., 2012).

Private Cloud is a cloud infrastructure made available only to organizational users or explicit clients and the infrastructure is managed by the organization themselves or third party providers. The adoption and deployment of this model is suitable for enterprises and government departments with couple of branches. Unlike public cloud, deployment of private cloud is mostly within enterprise environment, making system management a priority (Kahn et al., 2012).

Hybrid Cloud is a cloud infrastructure consisting of two or more cloud deployment types combined using special techniques that allow data and application transplant between them (Tanimoto et al., 2013).

Community Cloud is a cloud Infrastructure model particularly used for providing shared infrastructure 
between organisations and is managed by organisation them self or third party (Garlick. 2011).

\section{B. Cloud computing Services}

IaaS is a cloud service that enables users to remotely access cloud computing resources provisioned in cloud such as network, storage and processing. The resources are charged by pay per use and can be scalable depending on user demand, .e.g., Amazon EC2 and S3, Rack space Cloud, Microsoft, Terremark Enterprise cloud (Diez and Silva, 2013).

PaaS is used to offering an entire platform of computer, as well as design, testing, hosting and application development to users in their local system. Moreover, these services can be utilized to develop web application without users deploying any software or extra hardware facilities on their local computer. The key difference between SaaS and PaaS is SaaS fundamentally host mainly complete application whereas PaaS offers platform for application development, e.g., Google AppEngine, Facebook (Diez and Silva, 2013).

SaaS is a process where complete applications are hosted and offered on demand as a service over the internet. Users are not required to purchase or install software or application on their local computer rather by payment model, pay per use or order, through cloud provider. Moreover, this model eliminates totally issue of software maintenance or licensing. SaaS ensures rapid software deployment, thereby reducing cost of development and planning by organisation or end users e.g., SalesForce.com CRM, Google Mail, Google Doc (Fremdt et al., 2013).

\section{Cloud Computing Platform}

Cloud computing platform has tremendously evolved from recent advancement in virtualization technology. This has given rise to the utilization, enhancement and transformation to a more computational need in the form of cloud computing (Nwobodo et al., 2014). In this section, a comparison of four major cloud computing platform solution namely: Eucalyptus, Abicloud, OpenNebula and Nimbus will be presented.

\section{A. AbiCloud Platform}

AbiCloud computing platform is designed, developed by Abiquo; an established cloud computing company in Barcelona Spain specialized in development of cloud platform. Peng, et al, (2009), state that Abicloud computing innovation can be used to building, managing as well as integration in a homogenous environment a private and public cloud virtualized infrastructure. Moreover, the platform can enable user's automatically and easily scaling, orchestration, deployment of variety of cloud utilities, including management of storage system, servers, virtual devices, networks and application (Haji et al., 2010). These has potentiality of accomplishing IaaS services within a short time, and further leveraging such capability in delivering managed hosting services or self services IaaS via simplified interface or the combination of both.

AbiCloud web based management interface constitutes an exceptional classification in comparison to other cloud platform mainly designed to deploy services using command line process. Users have the ability to deploy new services such as virtual machines (VM) by drag and drop using mouse. Once deployment is completed, users can use RESTful API as well as other notable integration to connect all their services to their service desk, billing system, CMDB, customer portal, backup system, shopfront, OSS or BSS system.

The privileges, scopes and roles security mechanism on the platform provides opportunity to providers in delegating authority not just for task management but restriction of service desk staff and users to specific parts of the physical infrastructure and customer base.

The platform technology relieved users difficulties in building, developing and deploying cloud infrastructure by offering capabilities of entire plug and play solution. It maximises computing efficiency by introducing a simplified installation process that reduces disruption to organisation IT. AbiCloud platform can be used to manage EC2, facilitate deployment and implementation of hybrid or private cloud depending on user requirement, including easy deployment to another Abicloud platform. This can support different kinds of virtual machines e.g., VMware, KVM, vBox and is developed based on Java

AbiCloud VMS (Virtual Monitor System) is designed purposely to monitor virtual infrastructure primarily to study about status and events. AbiCloud WS is a virtual component part of the platform that communicates with all virtualized components principally responsible for management of all virtual machines. The Appliance Manager handles external application import to platform as well as enabling scalability, distribution and management. AbiCloud client component is a web application designed to permit management of private cloud by their respective owners (Haji et al., 2010). 


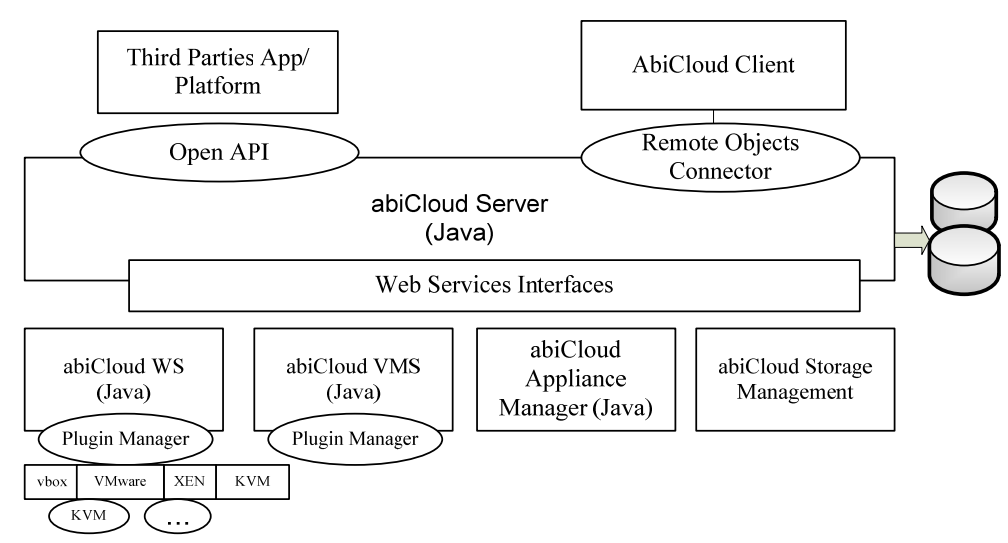

Figure 1. AbiCloud Platform (Peng et al., 2009).

\section{B. Eucalypus}

The Eucalyptus (Elastic Utility Computing Architecture for Linking Your Program to Useful Systems ). Eucalyptus program conceptually commenced in California University Santa Babara, specifically as an open source used to build private cloud platform. Currently, it has transformed and run by a Eucalyptus company (Zeng et al., 2012).

The platform is an open source implementation of Amazon EC2 \& S3 used to build private and hybrid cloud compatible with AWS, APIs and with minimum modification or extension can also support other clients. Eucalyptus is a designed platform that fulfils commercial need of EC2 cloud. It consists of a program called euca2ools, a front end for users, similar to EC2 program front end (Cordeiro et al., 2010; Sempolinski and Thain 2010).

Eucalyptus is structured as elastic computing that enable connection of users program to important system. The technology is an infrastructure that uses workstation and clusters to implement elastic, utility cloud computing and it is a well known service level protocol based standard computing that enable users provide lease computing network capability.

According to Wind (2011), Eucalyptus offers hierarchical platform structure, which encompasses an interface known as client API that enables connection and communication to each resource within the platform. Eucalyptus depicts three components as: Cloud Controller, Cluster Controller and Node Controller as shown in figure2 below (Haji et al., 2010). Cloud controller is responsible for resource information querying from node managers and then makes decision scheduling as well as using cluster controllers to execute them. Cluster controller task is collection of set of virtual machine information and on specified node controllers schedule their execution. Node controller runs in each of the node specifically designated to host virtual machine. All the inspection, implementation, as well as VM termination are managed in each host it runs on.

Eucalyptus users have the capacity to monitor and execute all virtual machine deployed in their environment in an easy, flexible modular fashion. The platform is designed to enable flexibility in moving applications to the platform as desired on public cloud. Moreover, it enables easy deployment on hybrid cloud using any resources available in either private or public cloud to its advantage. Path of its disadvantage is lack of interface for virtual machine management and advance monitoring (Haji et al., 2010).

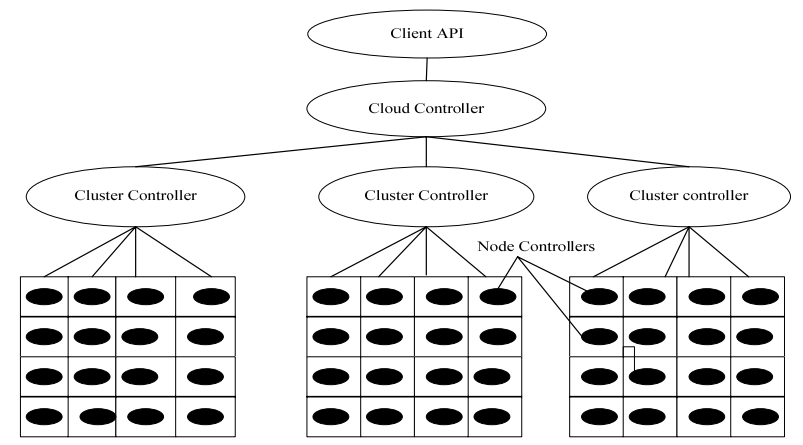

Figure 2. Eucalyptus Architecture (Haji et al., 2010).

\section{Nimbus}

Nimbus cloud platform is an integrated open set tool, used to deliver, providing infrastructure as a service cloud computing solution supported by University of Florida and Chicago (Zeng et al., 2012). Nimbus platform is specifically designed based on science community interest such as batch schedulers, proxy credentials and best efforts allocation etc., although it have recently supported non scientific applications. It enables users to provide, build various remote computing on demand resources via deployment of virtual machines. Nimbus permits combination of Amazon, OpenStack and many other clouds.

According to Wind (2011), Nimbus cloud seems more complicated than many cloud platforms as command and results are inputted in command lines, making nimbus appear extremely complex due to specific applications. Nevertheless, the software is particularly unique based on greater level of flexibility as it supports almost every hypervisors. The provisions on consistent and regular backup of platform workload help prevent issues of data loss in event of malfunction. Nimbus is supplied in apache version 2 licences like OpenNebula. Nimbus platform 
supports XEN and KVM virtualization technologies except VMware (Wu et al., 2010).

Peng, et al, (2009), states, Nimbus platform functional components can be grouped into three: client supported module, service supported module and resources management module. Client supported modules is used to support users especially with quick instance launches, one click clusters and consists of context client module, cloud client module, EC2 client module including reference client module. Service supported module is primarily used in providing various cloud services and the modules include EC2 WSDL module, remote interface module, and web service resource framework module. Background resource management module is principally used to manage a range of physical resources on cloud computing platform such as IaaS gateway module, work service management module, EC2 plus other supported platform module, workspace controller, workspace resource management module and workspace pilot module.

The workspace service module is a standalone or autonomous virtual machine manager whereby different remote protocol can access and invoke. The module is relevant to Java application while remains irrelevant to any content running on the system. Web service resource framework front end is the remote protocol implementation between client and workspace. EC2 front end is basically an implementation based remote protocol of web service description language (WSDL) for the platform of Amazon elastic cloud computing or SOAP and Query APIs that support EC2 client by allowing them develop nimbus at the same time EC2. Cloud client modules allow user an easy running of their respective operation by simple click action. All the characteristics of WSRF front are presented to users via reference module in command line way, making it complex as it consists of some application specific scripts (Wu et al., 2010; Peng et al., 2009).

Workspace pilot is a program that allows integration of VM s with previously configures resources to manage tasks. Typically, this is an optional module and the nodes deployed on the pilot program are not run but managed only by service program. Context agent module responds to client support to manage, coordinates large scale clusters auto start up services. Moreover, provide VM services and is capable of running both in nimbus platform including EC2 via backend services. Cumulus is a nimbus open source functional component of Amazon S3 REST API implementation. In addition, can be installed stand alone used as Nimbus repository solution.

EC2 gateway functionality ranges from scrutinizing the status of homogenous wireless sensor network, running the image of Amazon public cloud virtual machine on Amazon cloud platform, notifying users of the virtual machine public IP via the resource characteristics whenever it becomes available (Peng et al., 2009).

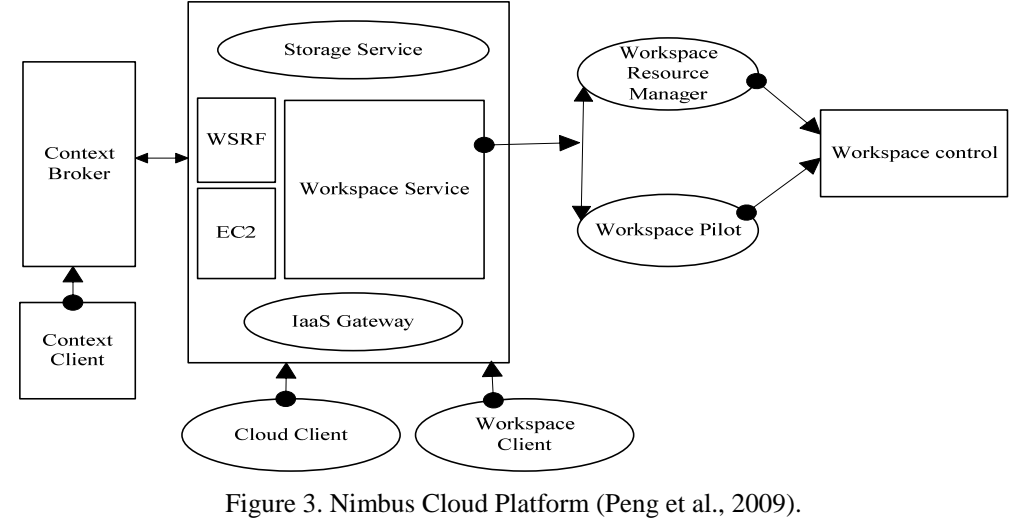

\section{Opennebula}

OpenNebula is a major cloud technology that evolves from many years of consistent research and development project by European Union in virtual machine efficiency, scalability, management on massive level distributed infrastructure. The platform is an open source cloud service. OpenNebula is an open source cloud computing tool specifically designed as modular to allow easy integration with different environment and hypervisors, further to manage the heterogeneity and complexity of distributed large infrastructure (Wen et al., 2012; Cordeiro et al., 2010).

The OpenNebula is a flexible, scalable, open virtual infrastructure mechanism, capable of enabling the synchronization of network, storage and virtual techniques. This can allow users to dynamically deploy services on distributed infrastructure based on provision strategies at data centre and remote cloud resources. Furthermore, OpenNebula facilitates cloud evolution by allowing users to integrate and leverages existing IT infrastructure to providing flexible, comprehensive, open and extensible management layer capable of orchestrating and automating enterprise cloud operation, in addition, deployment solution for storage, virtualization, networking, user management and monitoring can be achieved (Peng et al., 2009).

Users have the ability via the interior interface and OpenNebula data centre platform to deploy various kind of cloud. The platform is mainly used for management of data centre private cloud and cluster of infrastructures, including supports in connecting hybrid cloud to local and public infrastructure. OpenNebula can be used to build a cloud computing environment that is highly scalable. Moreover, public cloud is fully supported by provision of interfaces and vital functions to VMs, network and storage 
management. Users can access services via control interfaces provided by the platform. The key different of the platform with nimbus is the use of remote interface based on EC2 or WSRF by nimbus in which users can process entire security related cases which OpenNebula lacks such capability. (Peng et al., 2009),

The platform consists of three main components as shown in figure4 below such as: Core Virtual Infrastructure Manager, Capacity Manager and Drivers. The lifecycle of the virtual machine are managed with the Core Virtual Infrastructure Manager by ensuring smooth run of basic operations including migration, monitoring and deployment. The second component Capacity Manager (scheduler) module is principally responsible for managing all functionality from the Open Nebula core such as balancing workloads in virtual machines. The third component (“drivers") supports all platforms under it, because every necessary driver to regulate data transfer and to manage VM on all host, irrespective of the hypervisors are contained. (Peng et al, 2009). OpenNebula does not support Graphic User Interface (GUI) which is the main drawback to the platform. The platform provides good load balancing across nginx (Wind, 2011; Peng et al., 2009).

There are notable advantages of the platform such as server consolidation, partitioning dynamic clustering, centralized management of all workload, load balancing, heterogeneous workloads, especially virtual machine supply on demand. Part of the Open nebula advantages in terms of infrastructure management is the ability to dynamically adjust scale of cloud platform infrastructure by increasing the number of partition cluster and hosts in order to achieve various requirements. Another advantage is possibility of managing centrally entire distributed and physical infrastructure and ability to build at data centre infrastructure with heterogeneous resource. The resource integration process i.e. server integration, ensure resource efficiency by helping reduce demands or use of physical resource which further decrease cost of peak time requirement, cooling system, space, energy consumption etc. The OpenNebula scalability structure helps users meet rapid response demands.

The platform allows system integrators to deploy various clouds and integrate visual data centre services and products such as service manager, visual image manager, visual machine manager etc. The platform is a preferable choice in data centre because is an open source, extensible interface, flexible structure cloud solution. OpenNebula compared with Eucalyptus clouds has more power for private cloud support and dynamic, scalable, management of virtual machines on clusters. The platform in regard to hybrid cloud provides elastic mechanism and on demand resource access like Amazon EC2 (Wind, 2011; Peng et al., 2009).

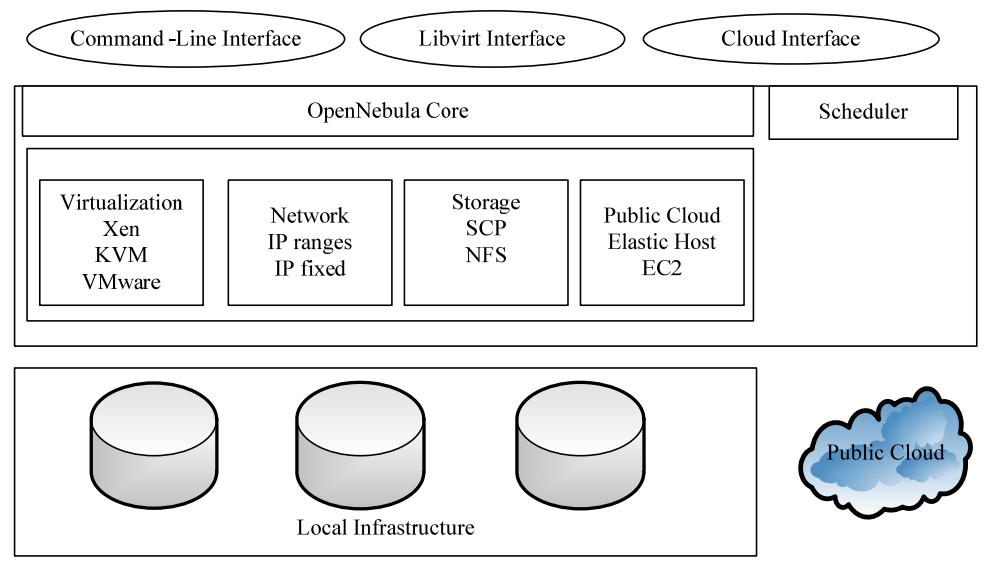

Figure 4. OpenNebula Platform (Haji et al., 2010).

\section{COMPARISON OF ClOUd COMPUTING PlATFORMS}

Presently, a lot of cloud computing platform exist with different implementation, characteristics and varying advantages. In order to have understanding of them, a comparison of four major platforms: Eucalyptus, OpenNebula, Abicloud and Nimbus cloud are presented in table I below and some parameters used in the analysis include: Platform type, cloud form, compatibility, deployment, deployment manner, Transplant ability, VM support, Web Interface, structure, Reliability, OS support and development. According to the table I, although most of the platforms are different in implementation, there are common similarities to some extent in many aspects. For an example, the whole platform supports IaaS services as well as scalability, dynamic platform deployment and Xen virtualization technology. Other areas of similarities are application development with Java and Linux operating system. However, they differ in a lot of ways in term of reliability, platform structure as well as interfaces of their network. In some instances some has a considerable advantage over others such as Abicloud which has outstanding flexible deployment process using GUI with mouse compared with others which can only be deployed using command line interface. In terms of reliability, 
TABle I. Cloud Platforms (Peng ET AL., 2009; KAhN ET AL., 2012).

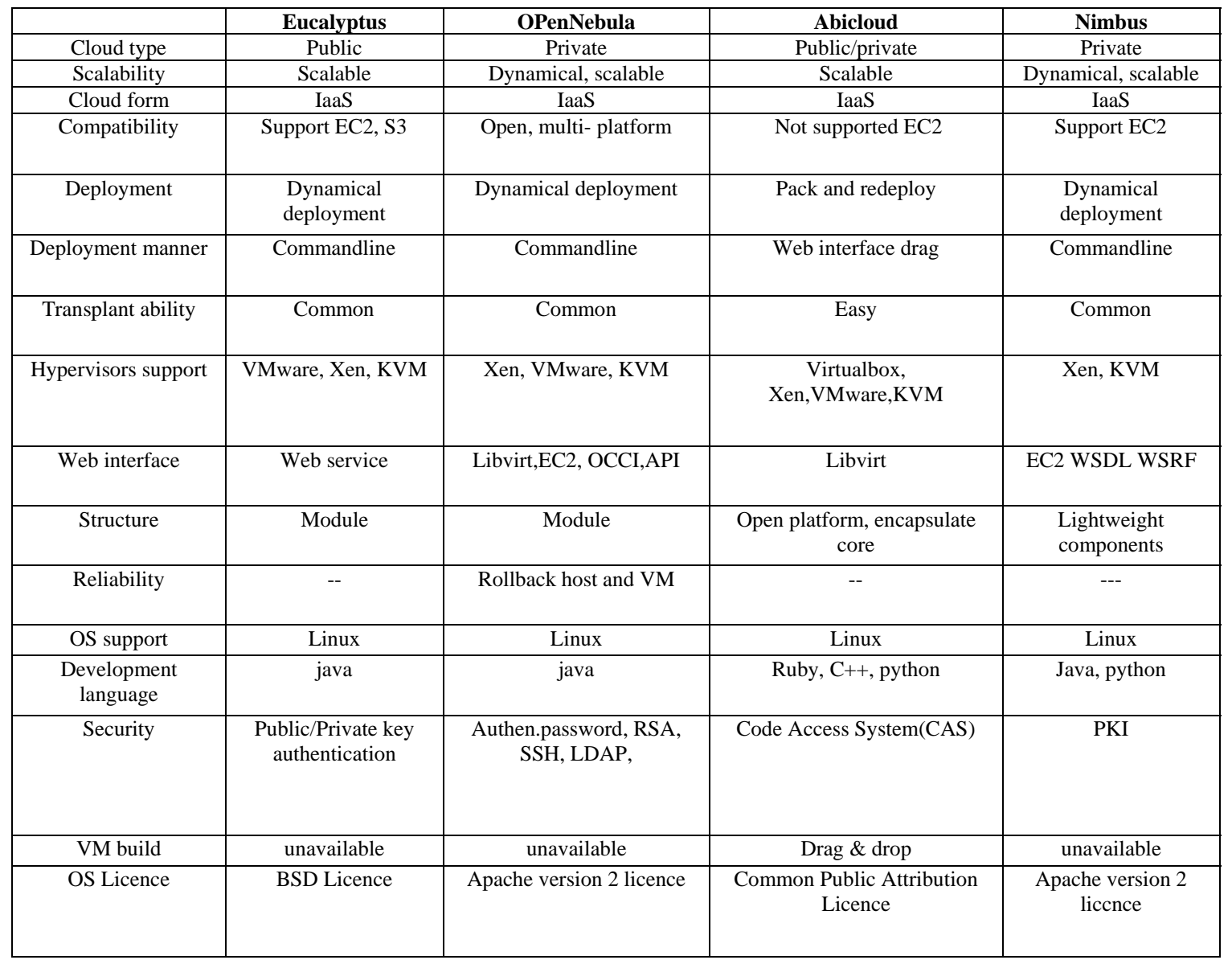

\section{Discussion ON CLOUd COMPUTING PlatForMS}

AbiCloud fundamentally seems robust for organization planning to build as well as integrating a more homogenous environment such as private and public cloud virtualized infrastructure. This is evidential considering the flexibility of their web based management interface which has capabilities such as deployment of various services especially virtual machine by drag and drop using mouse, RESTFul API and other integration for connecting Abicloud services etc.

The open nebula realistically is a preferable platform for organization expecting more of an efficient, scalable, dynamic management of VMs within a private cloud (data centre) reflecting the involvement of large entities of physical and virtual servers based on Hizea scheduling.

Retrospectively, Eucalyptus appears more suited to enterprises that require a private cloud based on organizational need and to operate a more protective strategies from users. This is achievable considering the potentiality of implementing or configuring multiple clusters within Eucalyptus, each having private internal network addresses, which can be transformed to a single cloud (private cloud).

Nimbus Cloud however seems suited to scientific cooperative community that is more interested in broad customization need rather than technical forms of the system. Globus Toolkit makes nimbus a reasonable choice for the scientific community due to their familiarity with the tool kit and the more conductive of excess cloud time sharing.

\section{CONCLUSION}

Cloud computing is a new internet based technology widely used and studied in recent memories. Currently, there are lots of cloud computing platform with varying level of application, architecture, characteristic etc. Now, the difference in the platform is becoming an issue in terms of understanding and usage. In this paper a detailed comparison of four major cloud computing platform has been presented. Based on the analysis, users now have the opportunity to understand the features and be able to make choices of cloud computing platform in respect to cloud 
computing module, services, development supports, cloud interfaces, deployment, OS supports and compatibility.

\section{REFERENCES}

[1] Ameen, M. N., Sanjay, H. A., \& Patel, Y. (2012, December). Nitte Meenakshi Inst. of Technol., Bangalore,

[2] India. In Parallel Distributed and Grid Computing (PDGC), 2012 2nd IEEE International Conference on (pp. 262-267). IEEE.

[3] Cordeiro, T., Damalio, D., Pereira, N., Endo, P., Palhares, A., Gonçalves, G., Sadok, D.; Kelner, J.; Melander,

[4] B.; Souza, V.; Mångs, J. E. (2010, November). Open source cloud computing platforms. In Grid and

[5] Cooperative Computing (GCC), 2010 9th International Conference on (pp. 366-371). IEEE.

[6] Diez, O., \& Silva, A. (2013). Govcloud: Using Cloud Computing in Public Organizations. Technology and Society Magazine, IEEE, 32(1), 66-72.

[7] Fremdt, S., Beck, R., \& Weber, S. (2013, January) Does Cloud computing matter? An analysis of the Cloud

[8] Model Software-as-a-Service and its impact on operational agility. In System Sciences (HICSS), 2013 46th Hawaii International Conference on (pp. 1025-1034). IEEE.

[9] Haji, A., Ben Letaifa, A., \& Tabbane, S. (2010, October). Cloud Computing: Several Cloud-oriented Solutions. In ADVCOMP 2010, the Fourth International Conference on Advanced Engineering Computing and Applications in Sciences (pp. 137-141).

[10] Garlick, G., "Improving Resilience with Community Cloud Computing," Availability, Reliability and Security (ARES), 2011 Sixth International Conference on, vol., no., pp.650, 655, 22-26 Aug. 2011. IEEE

[11] Peng, J., Zhang, X., Lei, Z., Zhang, B., Zhang, W., \& Li, Q. (2009, December) Comparison of several cloud computing platforms. In Information Science and Engineering (ISISE), 2009 Second International Symposium on (pp. 23-27). IEEE.

[12] Khalid A, Shahbaz M. CLOUD COMPUTING TECHNOLOGY: SERVICES AND OPPORTUNITIES. Pakistan Journal of Science [serial online]. September 2013; 65(3):348-351. Available from: Academic Search Complete, Ipswich, MA. Accessed June 20, 2014.

[13] Khan, N., Noraziah, A., Ismail, E. I., Deris, M. M., \& Herawan, T. (2012). Cloud computing: Analysis of various platforms.
International Journal of E-Entrepreneurship and Innovation (IJEEI), 3(2), 51-59.

[14] Lin, A., \& Chen, N. C. (2012). Cloud computing as an innovation: Perception, attitude, and adoption. International Journal of Information Management, 32(6), 533-540.

[15] NIST (2013) NIST Cloud Computing Standards Roadmap [online] http://www. boulder.nist.gov/itl/cloud/ up load/NIST_SP-500291_Version 2_2013_June 18_FINAL.pdf (accessed 30 July 2013).

[16] Nwobodo, I., Jahankhani, H., \& Edoh, A. (2014). 'Security challenges in the distributed cloud computing'. International Journal of Electronic Security and Digital Forensics, 6(1), 38-51.

[17] Sempolinski, P.; Thain, D., "A Comparison and Critique of Eucalyptus, OpenNebula and Nimbus," Cloud Computing Technology and Science (CloudCom), 2010 IEEE Second International Conference on, vol., no., pp.417, 426, Nov. 30 2010Dec. 32010

[18] Sultan, N. (2013). Cloud computing: A democratizing force?. International Journal of Information Management, 33(5), 810-815.

[19] Tanimoto, S., Sakurada, Y., Seki, Y., Iwashita, M., Matsui, S., Sato, H., \& Kanai, A. (2013, July). A Study of Data Management in Hybrid Cloud Configuration. In Software Engineering, Artificial Intelligence,

[20] Networking and Parallel/Distributed Computing (SNPD), 2013 14th ACIS International Conference on (pp. 381-386). IEEE

[21] Wen, X., Gu, G., Li, Q., Gao, Y., \& Zhang, X. (2012, May). Comparison of open-source cloud management

[22] platforms: OpenStack and OpenNebula. In Fuzzy Systems and Knowledge Discovery (FSKD), 2012 9th

[23] International Conference on (pp. 2457-2461). IEEE.

[24] Wind, S., "Open source cloud computing management platforms: Introduction, comparison, and

[25] recommendations for implementation," Open Systems (ICOS), 2011 IEEEConference on, vol., no., pp.175, 179, 25-28 Sept. 2011

[26] Wu, T. et al. (2010) A Survey of Open-Source Cloud Infrastructure using FutureGrid $\quad$ Testbed http://salsahpc.indiana.edu/b649proj/proj7.html(accessed 28 May 2014).

[27] Zeng, W. Zhao, J., \& Liu, M. (2012, July). Several public commercial clouds and open source cloud computing software. In Computer Science \& Education (ICCSE), 2012 7th International Conference on (pp. 1130-1133). IEEE. 